\title{
Application Strategy of Staged Nursing Intervention After the Surgery of Huge Rotator Cuff Tears Under the Arthroscopy
}

\author{
Hong Zhang, Liya Xu, Sujuan Lin, Xiaoyan Wang, Cuiqing Liu* \\ Department of Orthopedics, the First Affiliated Hospital, Jinan University, Guangzhou, China
}

Email address:

cuiqing.liu@yahoo.com (Cuiqing Liu)

${ }^{*}$ Corresponding author

\section{To cite this article:}

Hong Zhang, Liya Xu, Sujuan Lin, Xiaoyan Wang, Cuiqing Liu. Application Strategy of Staged Nursing Intervention After the Surgery of Huge Rotator Cuff Tears Under the Arthroscopy. American Journal of Nursing Science. Vol. 9, No. 4, 2020, pp. 225-230.

doi: 10.11648/j.ajns.20200904.20

Received: May 21, 2020; Accepted: June 4, 2020; Published: June 17, 2020

\begin{abstract}
Objective: To explore the application of staged nursing intervention after the surgery of huge rotator cuff tear under the arthroscopy, and to evaluate the impact on patients' postoperative rehabilitation. Methods: A total of 25 patients who underwent arthroscopic surgery for large rotator cuff tears in our hospital from January 2018 to December 2019 were selected. The intervention group used a staged nursing program to intervene, and Selected 25 patients who only received routine nursing intervention during the same period as the control group. The constant-murley shoulder function score, UCLA score, SF-36 quality of life score, and nursing satisfaction score were compared between the two groups after 6 months. Results: There was no statistically significant in the difference in the scores of pain, functional activity, shoulder joint mobility, and muscle strength of the constant-murley shoulder function score between the observation group and the control group before surgery $(P>0.05)$. The pain, functional activities, shoulder joint mobility and muscle strength scores of the observation group were significantly higher than those of the control group 6 months after surgery $(P<0.05)$, and the UCLA score and SF-36 score of the observation group 6 months after surgery were significantly higher than the control group $(P<0.05)$, and the nursing satisfaction score of the observation group was significantly higher than that of the control group $(P<0.05)$. Conclusion: Staged nursing intervention can significantly improve the Shoulder joint functional rehabilitation process and quality of life of patients who post operation of huge rotator cuff tears, and improve nursing satisfaction.
\end{abstract}

Keywords: WeChat, Continuing Care, Vitrectomy, Compliance, Eyesight

\section{Introduction}

Huge rotator cuff tear is a common shoulder joint disease. The rotator cuff is a general term for tendon tissue covering the subscapularis, supraspinatus, infraspinatus, and small round muscles covering the front, upper, and back of the shoulder joint. The rotator cuff is composed of four inner muscle tendons surrounding the shoulder joint to form a semi-circular aponeurotic structure. Its tendon fibers interweave and attach to the fibrous layer of the joint capsule and stop together in the groove of the upper half of the anatomical neck of the humerus. The four tendons keep the humeral head close to the glenoid, forming a sleeve-like structure that wraps around the humeral head and stops at the large and small nodules of the humerus, named for its shape resembling a cuff. The rotator cuff is the main structure to stabilize the shoulder joint. Huge rotator cuff tears refer to the tearing of 3 or more of the 4 tendons. When the rotator cuff is completely broken, the loss of its stabilizing effect on the humeral head will seriously affect the shoulder Joint abduction function and the patient's quality of life. Arthroscopic biceps patch surgery is a common method for surgical treatment of this disease. For patients with huge rotator cuff tears suitable for surgical treatment, biceps patch surgery should be performed as soon as possible. Although this surgical method is less invasive, the current nursing intervention for huge rotator cuff tears is a key part of the patient's postoperative functional rehabilitation. Effective 
nursing intervention can greatly promote the patient's postoperative recovery, shorten the patient's hospital stay, and promote early functional exercise of the affected limb. However, at present, there is no unified standard for nursing intervention for huge rotator cuff tears.

The shoulder pain in the elderly is mostly caused by a torn rotator cuff, and a considerable number of patients have a disease course of more than half a year. Chronic disease will lead to further fat infiltration of the torn rotator cuffs and retraction of the rotator cuffs, and the development of large rotator cuff tears, which makes the treatment of torn rotator cuffs still a challenge, which can also lead to poor prognosis or surgery Failed [1]. The literature has described several surgical techniques to repair huge rotator cuff tears, for example, partial repair techniques, latissimus dorsi transplantation, biceps brachii tendon transplantation (Chinese operation) [2], reverse shoulder joint replacement, Shoulder joint bridge patch technique and upper joint capsule reconstruction [3].

In recent years, biceps long head tendon transplantation (Chinese procedure) and upper joint capsule reconstruction have become the most used procedures for treating huge rotator cuff tears in our hospital. Reconstruction of the upper joint capsule is to place an autologous broad fascia as a graft under the acromion and the humerus head, replacing the supraspinatus tendon, giving the humerus head a fulcrum during the upward movement to restore the vertical force couple of the humeral head stability. Scholars such as Mihata [4] and Denard [5] have reported that the short-term clinical results of upper joint capsule reconstruction are satisfactory and can restore better stability of the humeral head.

Some scholars have reported that [6] the graft after reconstruction of the upper joint capsule has an important role in maintaining the stability of the glenohumeral joint and the biomechanical stability of the rotation center of the humeral head. Our hospital is one of the multi-center research units of the Huashan Hospital Professor Chen Shiyi's team using biceps long head tendon transplantation (Chinese operation). Our hospital also uses this operation to treat huge shoulder sleeve tears. This procedure has the same technical principle as the reconstruction of the upper joint capsule. This technique restores the stability of the glenohumeral joint and the humeral head by using the long head tendon of the biceps tendon. This technique can be used to perform a closer anatomical reconstruction without destroying the residual upper joint capsule and the deep layer of the rotator cuff. Some scholars reported that the preliminary results of this technique are satisfactory, and the clinical efficacy and clinical score have been improved [7].

This study aims to explore the application methods and effects of staged nursing intervention in the rehabilitation of patients with huge rotator cuff tears.

\section{Materials and Methods}

\subsection{Research Object}

A total of 25 patients who underwent arthroscopic surgery for huge rotator cuff tears in our hospital from January 2018 to December 2019 were selected as the observation group, and included in the standard: 1. Complete imaging examination before operation and diagnosed as a huge lacerated cuff tear; 2 . Treatment with arthroscopic repair; 3 . Obtain patient's informed consent and voluntarily participate in nursing intervention services, exclusion criteria: (1) patients with combined preoperative imaging examination showed severe osteoarthritis or rotator cuff joint disease; (2) patients with previous history of ocular trauma; (3) patients with a dislocation of shoulder joint or acromioclavicular joint; (4) patients with previous history of shoulder surgery; (5) patients with rheumatoid arthritis; (6) patients with incomplete clinical data or lost to follow-up. A total of 25 cases of the same cases undergoing routine nursing intervention during the same period were selected as the control group.

\subsection{Surgical Method}

All patients with huge rotator cuff tears were treated with biceps patch. The choice of surgical approach determines the location of the surgical incision and the tissue structure that passes through. The large rotator cuff tear is mainly repaired under arthroscopy.

\subsection{Nursing Method}

The main contents of nursing include admission education and management, operation period nursing, early postoperative rehabilitation nursing, and continuous rehabilitation nursing guidance. According to different surgical approaches and repair methods, targeted care, especially post-operative rehabilitation care and functional exercise, can greatly promote the recovery of patients' functions and shorten the patient's hospital stay. The control group used postoperative routine care, which mainly included admission guidance, simple health education, observation of the affected limbs, regular physical examination, prevention of complications, and guidance of patients on the use of pain medication. The observation group adopted stage nursing intervention as follows:

\subsubsection{Psychological Care}

Patients with huge rotator cuff tears have psychological problems due to sudden injuries, long-term pain, and role changes. Therefore, for patients with huge rotator cuff tears after biceps patch treatment, patients should be informed of the disease knowledge and treatment plan according to the specific situation of the patient to improve the patient's awareness of the disease, patiently listen to the patient's questions and Give answers to enable patients to be psychologically prepared for possible pain, complications and other sudden conditions, and maintain a positive attitude, so as to cooperate with treatment and rehabilitation exercises to speed up the rehabilitation process. For patients with severe anxiety and fear, arrange a psychologist to give them targeted guidance. 


\subsubsection{Stage Nursing}

(i). The Second Stage Rehabilitation Nursing

Rehabilitation nursing because the healing of tendons and bones takes 6-8 weeks, patients after rotator cuff repair should generally avoid active activities of rotator cuff muscles and muscle strength exercises within 6 weeks after surgery. During this period, patients should be trained for shoulder joint protection, including: After the patient's anesthesia fails, begin to perform palmar flexion and back extension, grasping fist to increase strength, adaptive functional exercise of left and right swing fist, and perform the above-mentioned training 2 to 3 times a day, each time 15 to 35 times. In addition, on the first day after the operation, the patient's shoulder of the patient's limbs can be started to train. During the training, pay attention to keeping the movement soft and slow, the range of motion is maintained at $140^{\circ}$ forward flexion, and the arm is outspread $60^{\circ}$, if the external rotation of the body side is $40^{\circ}$. From 2 to 6 weeks after surgery, you can perform hand elbow exercise, coldman exercise, long muscle contraction exercise, shrug resistance exercise, hand resistance shoulder blade exercise.

(ii). The Second Stage Rehabilitation Nursing

It is 6 to 12 weeks after the patient's operation. During this period, the patient can be strengthened for the shoulder joint and muscle, after the patient removes the abduction bracket, instruct the patient to enter the elbow shoulder, retract the shoulder, retract the back, upper limb rotation, abduction of the shoulder, and other strength and stretching exercises to maximize the patient's stretching and strength.

(iii). The Third Stage Rehabilitation Nursing

It is 13 weeks after the patient's operation. During this period, the patient's shoulder joint and muscle intensive rehabilitation exercise and resistance exercise are performed, including wall ladder exercise, dumbbell training, rowing or swimming exercise with both arms, Exercise with a hammer or dynamometer. The above exercise measures increase the active range of the shoulder joint, strengthen the muscle strength of the rotator cuff muscles and deltoid muscles in the scapula plane, and improve the coordination and flexibility of the shoulder joints in daily life. The above exercise measures increase the active range of the shoulder joint, strengthen the muscle strength of the rotator cuff muscles and deltoid muscles in the scapula plane, and improve the coordination and flexibility of the shoulder joints in daily life.

(iv). The Fourth Stage Rehabilitation Nursing

It is discharge guidance and follow-up. The recovery period of rotator cuff injuries after surgical treatment takes 6 months to 1 year. Rehabilitation care and exercise only rely on hospitalization. The rehabilitation effect is limited, and rehabilitation exercise outside the hospital also plays a vital role.

Therefore, when the patient is discharged from the hospital, a detailed rehabilitation training plan should be formulated for the patient, carefully explain the methods and precautions of the rehabilitation training, and conduct regular telephone follow-up to understanding the patient's recovery and ask the patient to return to the hospital for regular review. The treatment of huge rotator cuff tears with biceps patch is a surgical plan to relieve the pain of patients with huge rotator cuffs and restore the function of the affected limbs. The healing speed is relatively slow, and it takes a long time for rehabilitation and nursing cooperation. Professional and effective rehabilitation nursing intervention is an important factor to restore the function of the affected limb after operation. Based on the above research, it can be concluded that actively carrying out perioperative nursing and early postoperative rehabilitation nursing can greatly promote the rehabilitation of patients, shorten the hospitalization time of patients, and promote early functional exercise of affected limbs. Staged rehabilitation exercises and long-term rehabilitation nursing guidance after discharge from the hospital provide a powerful guarantee for the recovery of affected limb strength, joint function, and long-term efficacy.

\subsubsection{Early Eating and Nutrition Management}

(1) Shorten the time before eating and drinking: distribute enteral nutrition powder Ansu 1 to the patient as directed by the doctor before the operation, instruct the patient and his family how to prepare-Dissolve 6 flat spoons (nutrition powder configuration spoon) of Ansu powder in $200 \mathrm{ml}$ of warm boiled water to make $250 \mathrm{ml}$ of no residue nutrient solution, orally once before bedtime before surgery; on the day of surgery, follow the nurse's instructions and take another oral dose approximately 2 hours before the operation. (2) Early postoperative diet: Therefore, after the patient is evaluated for consciousness, vital signs and normal swallowing function, no nausea, vomiting, and willing to eat, the patient can be given a seated or semi-sitting position to assist with drinking water. No discomfort can guide patients to eat a small amount of food that is easy to digest. Observation of no discomfort can increase food intake gradually.

\subsubsection{Painless Management}

Adopting "multimodal analgesia", mainly including oral etoricoxib (Ankangxin) $120 \mathrm{mg}$ for advanced analgesia in the evening before surgery, Adopting "multimodal analgesia", mainly including oral etoricoxib (Ankangxin) $120 \mathrm{mg}$ for advanced analgesia in the evening before surgery, increase pain threshold, intraoperative intra-articular injection of analgesic, indwelling postoperative analgesic pump, combined use of intravenous analgesics, local ice compress, combined with topical buprenorphine analgesic sustained-release drug patch, oral Pain medicine and other measures to ensure that the patient's VAS score $\leq 3$ points during hospitalization, the pain is well controlled.

\subsection{Evaluation Index}

Before and 6 months postoperative follow-up, use the Constant-Murley shoulder function score from pain (total score 15 points), functional activity (total score 20 points), shoulder joint mobility (total score 40 points), muscle Evaluation of the improvement of shoulder joint function in 4 aspects (total score 25 points). The University of California at Los Angeles (UCLA) score evaluates shoulder joint function. The UCLA score is 0 to 35 . The higher the score, the better the 
shoulder joint function. The SF-36 quality of life scale was used to evaluate the quality of life before and 6 months after the operation. The average score was 100 points. The higher the score, the better the quality of life. Using the self-made nursing satisfaction score scale of our hospital to evaluate the satisfaction of nursing at 6 months after operation, the full score is 100 points, the higher the score, the higher the satisfaction.

\subsection{Statistical Method}

SPSS23.0 was used to analyze the data. The measurement data was expressed as mean \pm standard deviation. The mean of the two groups was compared using independent $t$ test. The count data was expressed as a percentage. The comparison used chi-square test. When the theoretical number $\mathrm{T}<1$ or $\mathrm{n}<$ 40, Fisher's exact test was used, and $P<0.05$ considered the difference to be statistically significant.

\section{Result}

\subsection{Comparison of General Information Between the Two Groups}

There was no statistically significant difference between the two groups of patients included in the comparison between the sex ratio, average age, injury time ratio ( $\geq 1$ year $/<1$ year), average operation time, whether the ratio was superior, and the ratio of Goutallier classification $(\mathrm{P}>0.05)$, as shown in Table 1 .

Table 1. Comparison of general information between the two groups $(n=25)$.

\begin{tabular}{|c|c|c|c|c|}
\hline Project & Observation group & Control group & Statistics & $\mathbf{P}$ \\
\hline Gender (Male /Female) & $11 / 14$ & $13 / 12$ & $\chi^{2}=0.231$ & 0.571 \\
\hline Age & $44.2 \pm 5.3$ & $43.9 \pm 5.7$ & $\mathrm{t}=0.193$ & 0.848 \\
\hline Injury time $(\geq 1$ year $/<1$ year $)$ & $8 / 17$ & $10 / 15$ & $\chi^{2}=0.347$ & 0.556 \\
\hline Operation time (min) & $67.4 \pm 5.6$ & $69.3 \pm 5.1$ & $\mathrm{t}=1.254$ & 0.216 \\
\hline Dominant hand (yes / no) & $17 / 8$ & $20 / 5$ & $\chi^{2}=0.936$ & 0.333 \\
\hline Goutallier classification (level I / level II) & $10 / 15$ & $12 / 13$ & $\chi^{2}=0.325$ & 0.569 \\
\hline
\end{tabular}

\subsection{Comparison of Shoulder Joint Function Score Between Two Groups of Patients}

Among the observation group and the control group, there were no statistically significant differences in the scores of preoperative pain, functional activity, shoulder joint mobility, and muscle strength (Table 2, $P>0.05$ ), the pain, functional activity, shoulder joint mobility, and muscle strength scores of the observation group were significantly higher than those of the control group at 6 months after surgery. There was no significant difference in UCLA score between the two groups of patients before operation $(P>0.05)$. The UCLA score of the observation group at 6 months after operation was significantly higher than that of the control group. As shown in Table 3.

Table 2. Comparison of Constant-Murley shoulder function score between two groups.

\begin{tabular}{|c|c|c|c|c|c|c|c|c|c|}
\hline \multirow[b]{2}{*}{ Group } & \multirow[b]{2}{*}{$\mathbf{n}$} & \multicolumn{2}{|l|}{ Pain } & \multicolumn{2}{|c|}{ Functional activity } & \multicolumn{2}{|c|}{ Shoulder joint mobility } & \multicolumn{2}{|c|}{ Muscle strength } \\
\hline & & Preoperative & $\begin{array}{l}6 \text { months } \\
\text { later }\end{array}$ & Preoperative & $\begin{array}{l}6 \text { months } \\
\text { later }\end{array}$ & Preoperative & $\begin{array}{l}6 \text { months } \\
\text { later }\end{array}$ & Preoperative & $\begin{array}{l}6 \text { months } \\
\text { later }\end{array}$ \\
\hline Observation & 25 & $10.3 \pm 2.2$ & $13.7 \pm 1.1$ & $14.8 \pm 2.5$ & $18.3 \pm 1.4$ & $25.7 \pm 2.4$ & $36.2 \pm 2.4$ & $16.4 \pm 2.7$ & $22.9 \pm 2.2$ \\
\hline Control & 25 & $10.1 \pm 2.4$ & $13.0 \pm 0.9$ & $14.7 \pm 2.6$ & $17.2 \pm 1.3$ & $26.1 \pm 3.1$ & $34.3 \pm 2.5$ & $16.8 \pm 3.2$ & $21.5 \pm 2.6$ \\
\hline $\mathrm{t}$ & & 0.307 & 2.463 & 0.139 & 2.879 & 0.510 & 2.741 & 0.478 & 2.055 \\
\hline $\mathrm{P}$ & & 0.760 & 0.017 & 0.890 & 0.006 & 0.612 & 0.009 & 0.635 & 0.045 \\
\hline
\end{tabular}

Table 3. Comparison of UCLA score between two groups of patients.

\begin{tabular}{llll}
\hline Group & n & Preoperative & 6 months later \\
\hline Observation & 25 & $12.5 \pm 3.1$ & $28.6 \pm 4.1$ \\
Control & 25 & $12.3 \pm 2.8$ & $26.1 \pm 4.2$ \\
$\mathrm{t}$ & - & 0.307 & 2.130 \\
$\mathrm{P}$ & - & 0.760 & 0.038 \\
\hline
\end{tabular}

\subsection{Comparison of Quality of Life and Nursing Satisfaction Between Two Groups of Patients}

There was no statistically significant difference in the SF-36 scores between the two groups before operation $(P>$ 0.05). The SF-36 score in the observation group was significantly higher than the control group at 6 months after operation, and the difference was statistically significant (Table 4, $P<0.05$ ), moreover, the nursing satisfaction score of the observation group was significantly higher than that of the control group, and the difference was statistically significant $(P<0.05)$, as shown in Table 4 .

Table 4. Comparison of quality of life and nursing satisfaction between two groups of patients.

\begin{tabular}{lllll}
\hline \multirow{2}{*}{ Group } & \multirow{3}{*}{ n } & \multicolumn{2}{l}{ SF-36 score } & $\begin{array}{l}\text { Satisfaction } \\
\text { score }\end{array}$ \\
\cline { 3 - 4 } & & Preoperative & June & $96.3 \pm 3.7$ \\
\hline Observation & 25 & $68.9 \pm 5.2$ & $89.7 \pm 4.1$ & $92.4 \pm 4.6$ \\
Control & 25 & $70.1 \pm 6.4$ & $85.0 \pm 3.9$ & 3.303 \\
t & - & 0.728 & 4.153 & 0.002 \\
P & - & 0.470 & $<0.001$ & \\
\hline
\end{tabular}




\section{Discussion}

Rehabilitation and care after the repair of huge rotator cuffs are very important. Surgery is only a small part of the treatment of large rotator cuff tears. Rehabilitation and nursing after the operation is a very important part for patients to achieve good clinical efficacy, relieve pain and restore function [8]. The clinical efficacy of huge rotator cuff tears is usually evaluated by comprehensive assessment such as life scale and pain score, but because China's clinical rehabilitation system is not as perfect as abroad. Many rehabilitation programs and rehabilitation training for patients with huge shoulder sleeve repairs are not based on common guidelines, nor are they all recovered in the same quality way. The surgical conditions of patients with huge rotator cuff tears vary greatly, such as the patient's age, course of disease, preoperative muscle strength, range of joint movement, and degree of osteoporosis. Whether it is possible to carry out early passive exercise after surgery, external fixation brace fixed time, individualized care, etc. need to be different from person to person, develop the best rehabilitation plan, design an accurate orthopedic rehabilitation nursing treatment plan to reduce postoperative The risk of tearing and optimizing the efficacy of postoperative rehabilitation [9].

Under the current medical conditions in mainland China, the nursing intervention for huge rotator cuff tears is still based on routine orthopedic disease nursing. Promotion model and good clinical efficacy. Some studies have reported that the intervention plan for rehabilitation nursing after repair of huge rotator cuff tears is based on the time node of tendon and bone healing [10], and is formulated according to the specific conditions of patients before and during surgery. Rehabilitation nursing program has good clinical efficacy. The main content of the staged nursing intervention in this study consists of rehabilitation training and nursing guidance, which not only provides a seamless link for postoperative painless rehabilitation, but also enhances the characteristic technology of continuing care in specialist nursing.

The formulation of a staged nursing intervention program requires first establishing adequate communication with the surgeon to understand and evaluate the patient's intraoperative conditions in detail, especially the tendon quality, osteoporosis, and tendon tension before and after repair and fixation. And this communication needs to continue throughout the rehabilitation follow-up process, which is an important part of achieving ultimately good clinical efficacy.

The first stage is the most critical and important stage. The rehabilitation goal of this stage is to advocate early passive tension-free activities under the premise of maximum protection, which can minimize pain and inflammation, and implement staged nursing interventions The nursing therapist needs to demonstrate hands-on, and teach the family members and patients to understand the importance of passive tension-free activities, and strictly educate patients on the importance of avoiding active lifting at this stage. The observation group of this study was significantly better than the control group in postoperative pain score. At the same time, the nursing therapist is also needed to help the patient learn to wear the shoulder brace correctly at this stage. The brace wearing time is determined according to the patient's specific rehabilitation nursing intervention plan, ranging from 6 to 12 weeks. The same rehabilitation nursing intervention can also encourage patients to hold the ball and squeeze immediately after waking up in order to promote the circulation of the upper limbs of the affected side as soon as possible after the operation.

The objectives of the staged rehabilitation nursing intervention in the second stage include restoring passive and active ranges of motion while activating pain, activating rotator cuff muscle strength, establishing a normal shoulder-brachial rhythm and restoring mild functional activities of daily life. In the observation group of this study, under the guidance of nursing therapists, their joint mobility and daily light activity scores were significantly higher than those of the control group. The third-stage rehabilitation intervention focuses on the further recovery of muscle strength, including antagonistic joint activity training and closed-loop joint exercise training. This stage of rehabilitation aims to further improve muscle strength for higher-level functional activities. When the fourth stage is successfully carried out, the nursing therapist needs to provide over-strength strength training, intensive closed-chain training, proprioception and physical fitness training according to the individual conditions of the patient's age, gender, daily life and outdoor activities. At this stage of the rehabilitation goal, not every patient can achieve a perfect state. The functional state and strength at this time after surgery, patients need to decide whether they are suitable or not suitable for the training intensity of this stage according to their own comprehensive physical fitness. Nursing therapists should also make decisions based on the individual situation of the patient, and should not only focus on the established functional requirements.

\section{Conclusion}

This study introduces a comprehensive rehabilitation program of staged nursing intervention. Compared with current routine nursing, our initial practice can significantly improve the clinical efficacy of patients with huge rotator cuff tears and can significantly improve the quality of life of patients after surgery. However, this study also has certain limitations. For example, only the short-term recovery period of 6 months after surgery is observed, the sample size is still small, but the new nursing intervention model that integrates nursing and rehabilitation still has certain promotion significance. In addition, large-scale and multi-center studies are needed to maintain clinical efficacy whether in the middle and long term after surgery.

\section{References}

[1] Oh JH, Kim SH, Kang JY et al. Effect of Age on Functional and Structural Outcome After Rotator Cuff Repair. Am J Sports Med 2010; 38: 672-8. 
[2] Achilleas, Boutsiadis, Shiyi et al. Long Head of the Biceps as a Suitable Available Local Tissue Autograft for Superior Capsular Reconstruction: "The Chinese Way". Arthroscopy Techniques 2017.

[3] Mihata T, Ph.D, Lee TQ et al. Clinical Results of Arthroscopic Superior Capsule Reconstruction for Irreparable Rotator Cuff Tears. Arthroscopy the Journal of Arthroscopic \& Related Surgery 2013; 29: 459-70.

[4] Mihata T, Mcgarry MH, Pirolo JM et al. Superior Capsule Reconstruction to Restore Superior Stability in Irreparable Rotator Cuff Tears: A Biomechanical Cadaveric Study. Am J Sports Med 2012; 40: 2248-55.

[5] Denard PJ, Brady PC, Adams CR et al. Preliminary Results of Arthroscopic Superior Capsule Reconstruction with Dermal Allograft. Arthroscopy: The Journal of Arthroscopic \& Related Surgery 2017: S0749806317310927.
[6] Adams CR, Demartino AM, Rego G et al. The Rotator Cuff and the Superior Capsule: Why We Need Both. Arthroscopy the Journal of Arthroscopic \& Related Surgery 2016; 32: 2628-37.

[7] Hufeland M, Wicke S, Verde PE et al. Biceps tenodesis versus tenotomy in isolated LHB lesions: a prospective randomized clinical trial. Archives of Orthopaedic and Trauma Surgery 2019.

[8] Kokmeyer D, Dube E, Millett PJ. Prognosis Driven Rehabilitation After Rotator Cuff Repair Surgery. Open Orthopaedics Journal 2016; 10: 339-48.

[9] Carnevale A, Longo UG, Schena E et al. Wearable systems for shoulder kinematics assessment: a systematic review. Bmc Musculoskeletal Disorders 2019; 20.

[10] Maximilian, Petri, Joshua et al. Arthroscopic Superior Capsule Reconstruction for Irreparable Rotator Cuff Tears. Arthroscopy Techniques 2015. 